XXXI International Workshop on High Energy Physics:

Critical Points in the Modern Particle Physics

International Journal of Modern Physics: Conference Series

Vol. 47 (2018) 1860090 (8 pages)

(C) The Author(s)

DOI: $10.1142 / \mathrm{S} 201019451860090 \mathrm{X}$

\title{
Einstein Equation for Nondual Field Matter Modifies Naiver-Stokes Dynamics
}

\author{
I. E. Bulyzhenkov \\ Moscow Institute of Physics 6 Technology and Lebedev Physics Institute RAS \\ 53 Leninsky Pros., Moscow, 119991, Russia \\ ibw@sci.lebedev.ru
}

Published 6 June 2018

\begin{abstract}
Cartesian extended matter has its own nondual analog of the 1915 Einstein Equation for pure field physics in nonempty moving space. This tensor balance of energy densities and local stresses leads to Maxwell-type equalities for inertial currents and vector geodesic equations for relativistic accelerations of the Ricci scalar for inertial and gravitational mass densities. Field inertia of slow energy flows reestablishes the living force feedback which is missed in Newton-Euler fluid dynamics and in the Navier-Stokes equation.
\end{abstract}

Keywords: Non-empty space; Extended matter; Cartesian physics; Inertial feedback.

\section{Introduction}

The concept of void space regions without matter was unclear to Descartes, who maintained the extension of matter or 'matter-extension' as the material space plenum, early discussed by Aristotle. Newton's successful dynamics of point-like masses shook the need in sophisticated matter-extensions with Cartesian vortex states. Nowadays, Newton's mechanics of localized masses and continuous gravitation fields in empty space forms the dual core of contemporary field theories. Not Cartesian physics, ${ }^{1}$ but Newtonian empty space modeling became mandatory for 1916 Einstein's gravitation in the weak field limit.

Dual classical physics for spatially separated matter and fields is traditionally assigned to the macroscopic world. At the same time, physicists tend to assign nondual material densities (of quantum fields) only to the microscopic world. But the unique physical reality is either dual or nondual regardless of the spatial scaling and mathematical formalisms in available theories. Similarly, the matter is either local or non-local regardless of suitable approaches to describe it. Now nonlocality

This is an Open Access article published by World Scientific Publishing Company. It is distributed under the terms of the Creative Commons Attribution 4.0 (CC-BY) License. Further distribution of this work is permitted, provided the original work is properly cited. 
of matter is reasonably considered beyond quantum physics. ${ }^{2}$ The world holism ideas and the global direct overlap of all material elements motivates our attempts to develop non-Newtonian mechanics for continuous mass densities of the extended particle. There is no in reality mesoscopic scales for reasonable transitions from nondual microscopic physics to the dual macroscopic model of Newton. Any material states should initially be considered in nondual field terms on micro-, macro-, and mega-scales at least in qualitative approaches, if not quantitative, to energy flows in the Universe.

The purpose of this note is to trace the balanced energy origin of extended sources in Einstein's metric gravitation, to study the strong field compensation of positive (kinetic) and negative (potential) self-energies of equal inertial and gravitational masses, to propose a nondual field analogue of the Einstein Equation, and to predict new laboratory dynamics of fluids for conceptual tests of Cartesian matterextension. Based on propositions of Aristotle, Descartes, $\mathrm{Mie}^{3}$ and Einstein, ${ }^{4}$ we shall try to study how to redesign classical relativistic gravitation in pure field terms without localized particles. Our initial idea is that 1938 Einstein-Infeld physics may coherently replace the supposed empty space (between the localized charges) with the Aristotle space plenum of moving material fields. This may assist to give up the unnecessary concept of Newton's point-like particles and to modify the old building of classical gravitation by strong field solutions without energy divergence and metric singularities in the center of gravitational sources.

The Einstein Equation in the dual (field + matter) Newtonian paradigm has been known since 1915. The similar tensor equation in the Cartesian paradigm of the united space-matter operates with a nondual field continuum of extended massenergies. This continuum is filled by overlapping radial vortexes with chaotic autorotations of elementary metric densities. Such permanent metric motions within the elementary Cartesian vortex reveal the kinematic origin of the mass-energy density $\mu c^{2}>0$. This active, kinetic energy density may be associated with the internal relativistic heat. The positive (kinetic, active) energy is always accompanied by equal, but negative (potential, passive) self-energy according to the principle of energy self-compensation (introduced below). There is no elementary inertial mass without chaotic auto-rotations of its metric space. And inertial charge densities $\sqrt{G} \mu$ with positive kinetic energy $\mu c^{2}$ generate self-gravitation with negative (passive) elementary energies of Cartesian vortex states. Integral computations result in elementary and global compensations of active and passive energies in the non-empty space Universe.

We will return to the Einstein idea that the geodesic equations of motion should follow from the strong-filed gravitation equation. Cartesian geodesic relations for Ricci material densities can be derived exactly from the nondual field analogue of the Einstein Equation. In order to demonstrate some practical benefits of non-empty space physics, we will modify the Navier-Stokes equation in terms of Ricci energy flows with the inertial feedback of the well known 'living forces', $-\mu \partial_{i} V^{2} / 2$. This 
dynamical pressure averts asymptotically divergent energy flows and can initiate conceptual tests of Newton vs Cartesian mechanics in the laboratory.

\section{1938 physics of pure fields for 1629 Cartesian vortex mechanics}

The predominant majority of people believe that electric charges and inertial masses are located within visible frames of macroscopic bodies. Nevertheless, the experiment is only a criterion of truth, but not the truth itself. Nonempty space of continuous material flows has been recognized by philosophers not only in the Ancient East and in Ancient Greece, but also by many contemporary thinkers in the West (Umov, Mie, Einstein, Infeld). "A coherent field theory requires that all elements be continuous... And from this requirement arises the fact that the material particle has no place as a basic concept in a field theory. Thus, even apart from the fact that it does not include gravitation, Maxwell's theory cannot be considered as a complete theory" as was stated by Einstein and Infeld ${ }^{4}$ in 1938 . Indeed, the postulated pointparticle paradigm results in Coulomb energy divergence, which terminates Classical Electrodynamics as a self-consistent theory. A point source in the Maxwell-Lorentz Equations may be considered as "an attempt which we have called intellectually unsatisfying" according to De Broglie. ${ }^{5}$ Einstein also criticized his 1915 field equation for the point gravitational source: "it resembles a building with one wing built of resplendent marble and the other built of cheap wood" (translation ${ }^{6}$ ).

The evolution of Einstein's theory of relativity has already passed three milestones. They are the 1905 postulates and the internal energy $m c^{2}$ of mechanical bodies, the 1915 geometrization of massless metric fields in the Einstein Equation under the Newton's empty space dogma, and the 1938 proposition to distribute particle's mass-energy $m c^{2}$ continuously over all spatial points of the material metric field in the non-Newton nondual approach to physical reality. Recall that the integration of particles into spatial structures of their fields was suggested by Einstein together with Infeld ${ }^{4}$ for the further evolution of all natural disciplines: "We would regard matter as being made up of regions of space in which the field is extremely intense... There would be no room in this new physics for both field and matter, for the field would be the only reality." However, the extended mass has not been yet adopted by modern relativists. Their Newtonian references traditionally associate General Relativity (GR) only with the 1916 empty space metric of Schwarzschild, later denied by Einstein for physical reality in the 1939 thought experiment. ${ }^{7}$ Researchers tend to use the Dirac delta-function for the formal presentation of the Lagrange material density $\mu(\mathbf{x})=m \delta(\mathbf{x}-\boldsymbol{\xi}) / \sqrt{\gamma}$ in the point mass action $S=-c \int m d s(\boldsymbol{\xi})=-c \iiint \int \mu(\mathbf{x}) d s(\mathbf{x}) \sqrt{\gamma} d^{3} x=-c \iiint \int \mu(\mathbf{x})\left(d s / \sqrt{g}_{o o} d x^{o}\right) \sqrt{\gamma g_{o o}} d^{4} x$ for the Newton empty space $x^{i}$ with material peculiarities along the path $\boldsymbol{\xi}\left(x^{o}\right)$.

The elementary mass density is to be a continuous spatial function in the Cartesian world and in the Einstein-Infeld pure field approach to matter. ${ }^{4}$ According to Einstein, there are equal inertial (active $\mu_{a}$ ) and gravitational (passive $\mu_{p}$ ) masses. Today the best mathematical candidate to match the sum of equal active and 


\section{E. Bulyzhenkov}

passive mass-energy densities in non-empty pseudo-Riemannian world is the Ricci scalar, ${ }^{8,9} R \equiv g^{\mu \nu} R_{\mu \nu}=\left(\mu_{a}+\mu_{p}\right) c^{2} / \zeta \varphi_{o}^{2}$. Hereinafter $\varphi_{o}=c^{2} / \sqrt{G}=1.04 \times 10^{27} V$ is the universal potential for the inertial/gravitational mass-energy charge $q_{m} \equiv$ $E_{m} / \varphi_{o}=\sqrt{G} m$ and $\zeta=1 / 8 \pi$. The symmetrical Ricci tensor $R_{\mu \nu}=\partial_{\lambda} \Gamma_{\mu \nu}^{\lambda}-$ $\Gamma_{\mu \rho}^{\lambda} \Gamma_{\nu \lambda}^{\rho}+\Gamma_{\mu \nu}^{\lambda} \partial_{\lambda} \ln \sqrt{g_{o o}}-\partial_{\mu} \partial_{\nu} \ln \sqrt{g_{o o}}$ was already found for the static metric of nonempty space, when $\sqrt{-g}=\sqrt{g_{o o}}, g_{o i}=0$, and $\partial_{o} g_{o o}=0$. Indeed, the distributed static mass-energy $M c^{2}$ warps $g_{o o}(r)=1 / g^{o o}(r)=\left[1-U_{o}(r) E_{p}^{-1}\right]^{-2}=\left(1+r_{o} / r\right)^{-2}$ for a probe mass-energy $E_{p}$ with the potential energy part $U_{o}(r)=-G M E_{p} / r c^{2}=$ $-r_{o} E_{p} / r$. Only two warped connections $\Gamma_{o o}^{i}=\partial_{i} g_{o o} / 2$ and $\Gamma_{i o}^{o}=\partial_{i} g_{o o} / 2 g_{o o}$, when $\partial_{o} g_{\mu \nu}=0$ and $U_{i}=0$, define $R_{o}^{o}=g^{o o} R_{o o}=g^{o o}\left(\partial_{i} \Gamma_{o o}^{i}-\Gamma_{o o}^{j} \Gamma_{o j}^{o}\right)=$ $\left[-\partial_{i}^{2} \ln \left(g_{o o}^{-1 / 2}\right)+\left(\partial_{i} \ln \left(g_{o o}^{-1 / 2}\right)\right)^{2}\right]$ and $R=g^{o o} R_{o o}+g^{i j} R_{i j}=g^{o o}\left(\partial_{i} \Gamma_{o o}^{i}-\Gamma_{o o}^{j} \Gamma_{o j}^{o}\right)$ - $\delta^{i j}\left(-\partial_{j} \Gamma_{o i}^{o}-\Gamma_{i o}^{o} \Gamma_{j o}^{o}\right)=2 R_{o}^{o}$. This geometrical formalism for static gravitational fields reveals the Ricci scalar meaning as a sum of passive and active mass densities,

$$
\frac{\varphi_{o}^{2} R(r)}{8 \pi}=\frac{\varphi_{o}^{2} R_{o}^{o}(r)}{4 \pi}=\frac{\vec{w}^{2}(r)}{4 \pi}+\frac{\left(-\varphi_{o}\right) \nabla \vec{w}(r)}{4 \pi} \equiv \mu_{a}(r) c^{2}+\mu_{p}(r) c^{2},
$$

where $\vec{w}(r)=-\nabla W(r), W=-\varphi_{o} \ln \left(1+r_{o} / r\right), \mu_{a}=\mu_{p}=m r_{o} / 4 \pi r^{2}\left(r+r_{o}\right)^{2}$, and $G_{o}^{o}=R_{o}^{o}-R / 2=0$.

Now we propose to develop Cartesian vortex dynamics on the basis of vortex mechanical fields $\varphi_{o} f_{\mu \nu} \equiv \varphi_{o}\left(\nabla_{\mu} u_{\nu}-\nabla_{\nu} u_{\mu}\right)$ from the local four-velocity $u_{\mu}=g_{\mu \nu} d x^{\nu} / d s$ of moving material space. Local mechanical and gravitational mass-energies originate from local vortex motion of this space, when $f_{\mu \nu} f^{\mu \nu} \neq 0$. Such vortex mechanical states are accompanied by the Principle of Equivalence of Einstein for active and passive masses and by the mutual compensation of active (kinetic, positive) and passive (gravitational, negative) self-energies. The relativistic invariant for the active (positive) mass-energy density of vortex mechanical space is $\left(-\varphi_{o}^{2} f_{\mu \nu} f^{\mu \nu} / 8 \pi\right)$, which is together with its passive partner (yin-yang analogy) is distributed continuously over all elementary 4-volumes $\sqrt{-g} d^{4} x \equiv \sqrt{g_{\text {oo }}} d x^{o} \sqrt{\gamma} d^{3} x$ in the action integral for the 'void nothing':

$$
\begin{array}{r}
S=-\frac{\varphi_{o}^{2}}{16 \pi c} \iiint \int\left(-f_{\mu \nu} f^{\mu \nu}+f_{\mu \nu} f^{\mu \nu}\right) d s \sqrt{\gamma} d^{3} x \equiv \\
-\frac{\varphi_{o}^{2}}{16 \pi c} \int\left(-f_{\mu \nu} f^{\mu \nu}+\frac{2 u_{\nu} \nabla_{\mu}\left(f^{\nu \mu} B\right)}{B}\right) B \sqrt{-g} d^{4} x-\frac{\varphi_{o}^{2}}{8 \pi c} \int \partial_{\mu}\left(\sqrt{-g} B u_{\nu} f^{\mu \nu}\right) d^{4} x .
\end{array}
$$

Here we introduced the kinematic scalar field $B(x) \equiv d s(x) / \sqrt{g_{o o}} d x^{o}$ next to the active and passive mass densities in the space-time elementary volume $\sqrt{-g} d^{4} x$,

$$
B(x) \equiv \frac{\sqrt{g_{\mu \nu} d x^{\mu} d x^{\nu}}}{\sqrt{g_{o o}} d x^{o}} \equiv \frac{1}{\sqrt{g_{o o}} u^{o}} \equiv \frac{\sqrt{1-\beta^{2}}}{1-g_{o i} v^{i} / \sqrt{g_{o o}}} \equiv \sqrt{1-\beta^{2}}\left(1+\frac{g_{o i} d x^{i}}{g_{o o} d x^{o}}\right) \text {. }
$$

This inertial field depends on the GR metric tensor $g_{\mu \nu}=g_{\nu \mu}$, the physical threevelocity $v^{i} \equiv c d x^{i} / \sqrt{g_{o o}} d x^{o}\left[1+\left(g_{o i} d x^{i} / g_{o o} d x^{o}\right)\right]$ and the relativistic speed factor 
$\beta^{2} \equiv \gamma_{i j} v^{i} v^{j} / c^{2}$, with the tensor $\gamma_{\mu \nu} \equiv g_{o \mu} g_{o \nu} g_{o o}^{-1}-g_{\mu \nu}$ for 3D and 4D manifolds. The inertial field $B(x)$ contributes to the four-current of mass-energy,

$$
J^{\mu}(x) \equiv 4 \pi c^{2} B \mu(x) u^{\mu} \equiv 4 \pi \mu(x) c^{2} \frac{d x^{\mu}}{\sqrt{g_{o o}} d x^{o}}=\varphi_{o}^{2} \frac{\partial_{\nu}\left(\sqrt{-g} B f^{\mu \nu}\right)}{\sqrt{-g}} .
$$

This Maxwell-type equation for mass-energy currents is the equality due to the Einstein equality of active and passive mass densities. The variational equation (4) supports the complex charge $Q=\operatorname{Re} Q+i \operatorname{Im} Q$ for the double unification ${ }^{10}$ of particles with fields and gravity with electricity, when the internal energy of restframe charges is always complex, $E_{Q} \equiv Q \varphi_{o} \Rightarrow i\left(e_{o}-i \sqrt{G} m_{o}\right) \varphi_{o}=0,511 M e V+$ $1,04 i \times 10^{21} \mathrm{MeV}$ for the electron.

By applying the Einstein Principle of Equivalence to moving densities of active and passive masses in (2), one can say from (4) that the mass density $\mu(x)$ of extended matter in Cartesian mechanics originates from vortex velocities of material space:

$$
\begin{aligned}
\mu(x) & =-\frac{\varphi_{o}^{2}\left(\partial_{\mu} u_{\nu}-\partial_{\nu} u_{\mu}\right)\left(\nabla^{\mu} u^{\nu}-\nabla^{\nu} u^{\mu}\right)}{8 \pi c^{2}} \Rightarrow-\frac{m\left(\partial_{i} u_{o}-\partial_{o} u_{i}\right)\left(\nabla^{i} u^{o}-\nabla^{o} u^{i}\right)}{4 \pi r_{o}} \\
& =-\frac{m\left(\partial_{i} \sqrt{g_{o o}}\right)\left(g^{i j} \partial_{i} u^{o}+g^{i j} \Gamma_{i o}^{o} u^{o}-g^{o o} \Gamma_{o o}^{i} u^{o}\right)}{4 \pi r_{o}} \\
& =\frac{m \delta^{i j} \partial_{i} g_{o o} \partial_{j} g_{o o}}{16 \pi r_{o} g_{o o}^{2}}=\frac{m r_{o}}{4 \pi r^{2}\left(r+r_{o}\right)^{2}} .
\end{aligned}
$$

General relations (4)-(5) can be applied to one elementary (radial) particle without net rotations in its rest frame in order to derive the $\mathrm{known}^{8}$ relations for the elementary mass density $\mu(r) \Rightarrow m n(r)=m r_{o} / 4 \pi r^{2}\left(r+r_{o}\right)^{2}$, where $r_{o} \equiv \sqrt{G} m / \varphi_{o}=$ $m c^{2} / \varphi_{o}^{2}$ and $u_{o}=1 / u^{o}=\sqrt{g_{o o}}, g_{o o}(r)=r^{2} /\left(r+r_{o}\right)^{2}, \gamma_{i j}(r)=\delta_{i j}, g^{i j}(r)=-\delta^{i j}$, $\sqrt{-g}=\sqrt{g_{o o}}$. Indeed, $B=1$ in this frame of reference and $4 \pi r_{o} n(r) u_{o}=$ $g^{\nu \lambda} \nabla_{\lambda}\left(\partial_{o} u_{\nu}-\partial_{\nu} u_{o}\right)=-g^{i j} \nabla_{j} \partial_{i} u_{o}=\delta^{i j}\left(\partial_{j} \partial_{i} u_{o}-\Gamma_{o j}^{o} u_{o}\right)=r_{o}^{2} / r\left(r+r_{o}\right)^{3}$ or $n(r)=$ $r_{o} / 4 \pi r^{2}\left(r+r_{o}\right)^{2}$. Similarly for contrvariant mass-energy currents, $4 \pi r_{o} n(r) u^{o} \sqrt{-g}=$ $4 \pi r_{o} n(r)=\partial_{i}\left[\sqrt{-g}\left(\nabla^{o} u^{i}-\nabla^{i} u^{o}\right)\right]=\partial_{i}\left[\sqrt{g_{o o}}\left(g^{o o} \Gamma_{o o}^{i} u^{o}-g^{i j} \partial_{j} u^{o}-g^{i j} \Gamma_{j o}^{o} u^{o}\right)\right]$ $=\partial_{i}\left[\sqrt{-g_{o o}}\left(-g^{i j}\right)\left(g^{o o} \partial_{j} g_{o o}+\partial_{j} u^{o}\right)\right]=\delta^{i j} \partial_{i} \partial_{j} \ln \sqrt{g_{o o}}=r_{o}^{2} / r^{2}\left(r+r_{o}\right)^{2}$.

Despite inertial (kinetic) and gravitational (potential) mass densities are equal in (1) and (3), the positive kinetic $\left(+\mu_{a} c^{2}\right)$ and the negative potential self-energies have opposite signs. The gravitational self-action always arises due to the intense interaction potential ${ }^{8-10} W(r) \equiv \varphi_{o} \ln \sqrt{-g}=\varphi_{o} \ln \sqrt{g_{o o}(r)}=-\varphi_{o} \ln \left[\left(r+r_{o}\right) / r\right]$ of the emerged charge density $\sqrt{G} \mu_{p}$, with $\mu_{p} \equiv \mu_{a}$ :

$$
E_{k i n}+U_{\text {self }}=\int\left[\mu_{a}(r) c^{2}-\sqrt{G} \mu_{p}(r) \varphi_{o} \ln \frac{1}{\sqrt{g_{o o}(r)}}\right] \sqrt{\gamma} d^{3} x=\left(m_{a}-m_{p}\right) c^{2} \equiv 0 .
$$

Here the joint energy density in brackets is negative only in the very core of the radial carrier and is positive for all radial distances $r>r_{o} /(e-1)$. The integral energy balance (6) means the exact mutual compensation of kinetic and potential 
self-energies of any rest-mass body due to the Einstein Principle of Equivalence for inertial $\left(m_{a}\right)$ and heavy $\left(m_{p}\right)$ masses. Contrary to measurable exchanges of internal and translation kinetic energies, the negative potential energy $\left(-m_{p} c^{2}\right)$ is not relevant to observations in practice. But negative energy tensions within the continuous carrier (of the elementary relativistic heat $Q=m c^{2}$ ) is relevant to stability of the elementary extended mass-energy $m_{a} c^{2}$ by the internal negative pressure, once assumed by Anri Poincare for the extended particle. The same zero energy balance is true for active and passive electric (imaginary) energies of the extended electron. ${ }^{10}$ Like in the Terletsky quadriga, one isolated electron is already the zero sum carrier of passive/active mechanical and electrical energies, rather than only the electron - positron pair plus their supposed co-partners with unphysical negative masses.

\section{Nondual Einstein-type equation for moving material space}

The last integral in the 'void nothing' action (2) can be replaced by integration of 4-forces $u_{\nu} f^{\mu \nu} B$ over the hyper-surface that provide the internal Poincare pressure for stabilization of the extended mass. The other part of the action integral is related to active and passive mass densities, which we describe through the Ricci scalar R,

$$
\mu(x) \equiv \frac{\varphi_{o}^{2} R(x)}{16 \pi c^{2}}=\frac{\mu_{a}(x)+\mu_{p}(x)}{2}=-\frac{\varphi_{o}^{2} f_{\mu \nu} f^{\mu \nu}}{16 \pi c^{2}}+\frac{\mu u_{\mu} u^{\mu}}{2} .
$$

Now we perform the Hilbert metric variations over $\delta g^{\mu \nu}$ in $(2), \delta S=-\left(\varphi_{o}^{2} / 16 \pi c\right) \int d^{4} x \delta(\sqrt{-} g B R)-\left(\varphi_{o}^{2} / 8 \pi c\right) \int d^{4} x \partial_{\mu} \delta\left(\sqrt{-g} B u_{\nu} f^{\mu \nu}\right)$ $\equiv \int \sqrt{-g} d^{4} x T_{\mu \nu} \delta g^{\mu \nu} / 2 c=0$, in order to derive a nondual analog of the Einstein Equation for pure field physics of moving material flows,

$$
T_{\mu \nu} \equiv \zeta \varphi_{o}^{2}\left[\left(u_{\mu} u_{\nu}-\gamma_{\mu \nu}\right) \frac{B R}{2}-B R_{\mu \nu}+\nabla_{\mu} \nabla_{\nu} B-g_{\mu \nu} \nabla_{\lambda} \nabla^{\lambda} B\right]+P_{\mu \nu} \equiv 0 .
$$

The Ricci material flows of active and passive mass densities are balanced in (8) by the Poincare negative stress $P_{\mu \nu}$, which originates from the Hilbert variations of the local force densities $\delta\left(\sqrt{-g} B u_{\nu} f^{\mu \nu}\right) / \delta g^{\mu \nu}$ in (2).

Empty space regions in Newtonian dual physics are described by field solutions with zero Ricci curvatures, $R_{\mu \nu}=0$. The motion of point material peculiarities in such massless fields can be approximated only through successive iterations. ${ }^{11,12}$ Cartesian physics of material fields is free from these complicated iteration procedures. One can find the exact geodesic equations for any non-equilibrium motion of inertial Ricci densities in the world metric space-time as the vector flow conservation from the general tensor balance (8), $\nabla_{\nu} T_{\mu}^{\nu}=0$. Here it is sufficient to make use of the known geometrical equalities $\nabla_{\nu} \nabla_{\mu} \nabla^{\nu} B-\nabla_{\mu} \nabla_{\nu} \nabla^{\nu} B \equiv R_{\mu \nu} \nabla^{\nu} B \equiv R_{\mu}^{\nu} \nabla_{\nu} B$ and the Bianch identities $2 \nabla_{\nu} R_{\mu}^{\nu} \equiv \nabla_{\mu} R$ :

$$
\frac{\varphi_{o}^{2}}{16 \pi}\left[\nabla_{\nu}\left(B R u_{\mu} u^{\nu}\right)-B \nabla_{\mu} R-\gamma_{\mu \lambda} \nabla^{\lambda}(B R)\right]=-\nabla_{\nu}\left(g^{\nu \lambda} P_{\mu \lambda}\right) .
$$


This general equation of motion can be equally read for the 4-acceleration $u^{\nu} \nabla_{\nu} u^{\mu}$ along a normal axis to the 4 -velocity $u^{\mu}$ of the scalar mass density $\mu=\varphi_{o}^{2} R / 16 \pi c^{2}$,

$$
B \mu c^{2} u^{\nu} \nabla_{\nu} u^{\mu}+\left(u^{\mu} u^{\nu}-g^{\mu \nu}\right)\left[\frac{g_{o \nu}}{g_{o o}} \nabla_{o}(\mu B)-\mu \nabla_{\nu} B\right] c^{2}=\left(u^{\mu} u^{\nu}-g^{\mu \nu}\right) \nabla_{\lambda} P_{\nu}^{\lambda}
$$

The vector equation (10) for Cartesian mechanics claims that the inertial field $B(x) \neq$ const contributes to geodesic dynamics of material space densities, while dual theories with the massless space alternative, where $B=$ const, are free from such kinematic feedbacks.

\section{Modified Navier-Stokes equation from geodesic flows of pure field energy}

The Cartesian acceleration law (10) for mass-energy flows within the Einstein-Infeld material space can be considered in the limit of vanishing metric fields, $g_{\mu \nu} \rightarrow \eta_{\mu \nu} \equiv$ $\{1,-1,-1,-1\}$, and low speeds, $\beta^{2} \rightarrow 0, B \rightarrow 1-\beta^{2} / 2, v^{i} \rightarrow c d x^{i} / d x^{o} \equiv V^{i}=V_{i}$, $d V^{i} / d t=\partial_{t} V^{i}+V^{j} \partial_{j} V^{i}=\partial_{t} V^{i}+\delta^{i j} \partial_{j} V^{2} / 2-[\mathbf{V} \times \operatorname{curl} \mathbf{V}]^{i}$ :

$$
\mu\left(\partial_{t} V^{i}+V^{j} \partial_{j} V^{i}\right)+V^{i} \partial_{t} \mu+\frac{\mu \partial_{i} V^{2}}{2}=-\partial_{i} p+\mu \nu \partial_{j} \partial^{j} V^{i}+\sum_{k=1}^{n} f_{k}^{i} .
$$

Here the post Euler term $V^{i} \partial_{t} \mu$ is the 1903 Tsiolkovsky force for the reactive rocket motion. The new kinematic feedback $\mu \partial_{i} V^{2} / 2$ originates from gradients of the scalar inertial field (3), which is not relevant to point-mass dynamics in Newtonian empty space. The restraining positive potential $V^{2} / 2$, which modifies inertia of Euler's fluids and the Navier - Stokes dynamics, appears in (11) exclusively in Cartesian physics due to the nondual tensor equation (8) for the nonempty inertial space. The right-hand side of (11) is associated with accelerating forces from the stress tensor $P_{\mu}^{\nu}$ in (10). Here we underlined the Euler's equation force with the pressure $p$ gradient and the simplest drag force with the Stokes kinematic viscosity $\nu$ next to all other possible forces $\sum f^{i}$, which can drive fluids out of equilibrium. New low speed equation (11) predicts in practice the measurable pressure gradient, $\partial_{r} p(r)=$ $-\mu \partial_{r} V^{2}(r) / 2$, across radial sections of tubes with stationary fluid or gas flows. Contrary to (11), the conventional Navier-Stokes equation provides $\partial_{r} p(r)=0$ in tubes that enables laboratory tests "Newton vs Descartes".

\section{References}

1. D. Garber, Descartes's Metaphysical Physics (University of Chicago Press, Chicago, 1992)

2. S. Popescu, Nonlocality beyond quantum mechanics. Nature Physics 10, 264-270 (2014)

3. G. Mie, Grundlagen einer Theorie der Materie. Ann. der Physik.37, 511-534 (1912); ibid 39, 1-40 (1912); 40, 1-65 (1913)

4. A. Einstein, L. Infeld, The Evolution of Physics (Cambridge Press, Cambridge, 1938)

5. L. de Broglie, New perspectives in physics (Basic Books, New York, 1962)

6. M.-A. Tonnelat, The Principles of Electromagnetic Theory and Relativity (Riedel Publishing Co., Dordrecht, 1966) 
7. A. Einstein, On a Stationary System With Spherical Symmetry Consisting of Many Gravitating Masses. Annals of Mathematics 40, 922-936 (1939)

8. I.E. Bulyzhenkov, Einstein's Gravitation for Machian Relativism of Nonlocal EnergyCharges. Int. Jour. of Theor. Phys. 47, 1261-1269 (2008)

9. I.E. Bulyzhenkov, Geometrization of Radial Particles in Non-Empty Space Complies with Tests of General Relativity. Jour. Mod. Phys. 3, 1465-1478 (2012)

10. I.E. Bulyzhenkov, Complex charge densities unify particles with fields and gravitation with electricity. Bulletin Lebedev Phys. Inst, 43, 138-142 (2016); EPJ Web of Conferences 126, 04008 (2016)

11. A. Einstein, L. Infeld, \& B. Hoffmann, The Gravitational Equations and the Problem of Motion. Ann. Math.39, 65-100 (1939)

12. A. Einstein, L. Infeld, On the motion of particles in general relativity theory. Canad. Jour. Math. 1, 209-241 (1949) 